\title{
The COPD assessment test: a systematic review
}

\author{
Nisha Gupta ${ }^{1,2}$, Lancelot M. Pinto ${ }^{1}$, Andreea Morogan ${ }^{1}$ and Jean Bourbeau ${ }^{1,2}$
}

Affiliations: ${ }^{1}$ Respiratory Epidemiology and Clinical Research Unit, McGill University Health Centre, McGill University, Montréal, QC, Canada. ${ }^{2}$ Dept of Epidemiology, Biostatistics and Occupational Health, McGill University, Montréal, QC, Canada.

Correspondence: Jean Bourbeau, Respiratory Epidemiology and Clinical Research Unit, Montreal Chest Institute, 3650 Rue St-Urbain, Office K1.32, Montréal, QC, H2X 2P4, Canada.

E-mail: jean.bourbeaudamcgill.ca

ABSTRACT The COPD assessment test (CAT) is a self-administered questionnaire that measures healthrelated quality of life. We aimed to systematically evaluate the literature for reliability, validity, responsiveness and minimum clinically important difference (MCID) of the CAT.

Multiple databases were searched for studies analysing the psychometric properties of the CAT in adults with chronic obstructive pulmonary disease. Two reviewers independently screened, selected and extracted data, and assessed methodological quality of relevant studies using the COSMIN checklist.

From 792 records identified, 36 studies were included. The number of participants ranged from 45 to 6469 , mean age from 56 to 73 years, and mean forced expiratory volume in $1 \mathrm{~s}$ from $39 \%$ to $98 \%$ predicted. Internal consistency (reliability) was $0.85-0.98$, and test-retest reliability was $0.80-0.96$. Convergent and longitudinal validity using Pearson's correlation coefficient were: SGRQ-C 0.69-0.82 and 0.63, CCQ $0.68-0.78$ and 0.60 , and mMRC $0.29-0.61$ and 0.20 , respectively. Scores differed with GOLD stages, exacerbation and mMRC grades. Mean scores decreased with pulmonary rehabilitation (2.2-3 units) and increased at exacerbation onset (4.7 units). Only one study with adequate methodology reported an MCID of 2 units and 3.3-3.8 units using the anchor-based approach and distribution-based approach, respectively. Most studies had fair methodological quality.

We conclude that the studies support the reliability and validity of the CAT and that the tool is responsive to interventions, although the MCID remains debatable.

@ERSpublications

Studies support the reliability, validity and responsiveness of the CAT as a HRQoL tool but its MCID remains unclear http://ow.ly/xkVNA

For editorial comments see page 833 .

This article has supplementary material available from erj.ersjournals.com

Received: Feb 052014 | Accepted after revision: May 222014 | First published online: July 032014

Support statement: This research was supported by a studentship award from the Research Institute of the McGill University Health Centre (Graduate Student Scholarship award to Nisha Gupta), the Collaborative Innovative Research Fund (CIRF) GSK Canada, the Canadian Cohort Obstructive Lung Disease (CanCOLD) Canadian Institutes of Health Research (CIHR)/Rx\&D Collaborative Research Program (IRO-93326), and the Respiratory Health Network of the Fonds de recherche du Québec - Santé (FRQS).

Conflict of interest: Disclosures can be found alongside the online version of this article at erj.ersjournals.com

Copyright @ERS 2014 


\section{Introduction}

Establishing a diagnosis of chronic obstructive pulmonary disease (COPD) requires spirometry; however, recent guidelines suggest that classifying COPD solely by forced expiratory volume in $1 \mathrm{~s}$ (FEV1) \% predicted is inadequate in reporting disease severity [1]. Assessing a patient's health-related quality of life (HRQoL) allows clinicians to make individualised patient management decisions; thus, the Global Initiative for Chronic Obstructive Lung Disease (GOLD) strategy document advocates that COPD management no longer be stratified solely by spirometric classification, but through a multidimensional assessment of specific patient attributes $[2,3]$.

COPD-specific questionnaires assessing HRQoL do exist (e.g. the St George's Respiratory Questionnaire (SGRQ) or the Chronic Respiratory Questionnaire (CRQ)), although some are impractical for clinical use as they are time consuming [2]. GOLD consequently proposes using either the modified British Medical Research Council (mMRC) dyspnoea scale or the COPD assessment test (CAT); however, preferential recommendation is given to the CAT since it provides a thorough coverage of the impact of COPD on wellbeing [2].

The CAT was created using COPD patients' input, then developed using modern questionnaire methodology: psychometric analysis and item response theory using Rasch analysis identified items with the best fit to form a unidimensional instrument $[4,5]$. The self-administered questionnaire consists of eight items assessing various manifestations of COPD aiming to provide a simple quantified measure of HRQoL [5]. A preliminary evaluation of the CAT's psychometric properties has been promising [5]. Summarising the current knowledge on the performance of this tool as a HRQoL measurement instrument is valuable, as the test could have important roles in COPD clinical practice and research. To the best of our knowledge, a comprehensive review of the psychometric properties of the CAT questionnaire has not been conducted.

Our objectives for this review were to systematically search the literature to evaluate and summarise the psychometric properties of the CAT (reliability, validity, responsiveness and minimum clinically important difference (MCID)) as a HRQoL instrument used in patients with COPD.

\section{Methods}

Detailed descriptions of the psychometric properties assessed in the review and the completed Preferred Reporting Items for Systematic Reviews and Meta-Analyses (PRISMA) checklist can be found in the online supplementary material.

\section{Eligibility criteria for study selection}

Randomised controlled trials and observational studies (e.g. cross-sectional, cohort, etc.) with $>10$ participants were included. Study participants needed to be subjects aged $\geqslant 40$ years diagnosed with COPD (using the GOLD criteria) [2]. Interventions could have been any intervention, placebo, usual care or time. Outcomes evaluated consisted of the CAT reliability, validity, responsiveness or MCID. Studies that reported at least one or more psychometric properties were included. Detailed inclusion and exclusion criteria can be found in the online supplementary material.

\section{Information sources and search}

A search was conducted on March 10, 2014 in the Cochrane Database of Systematic Reviews, the Database of Abstracts of Reviews of Effects, and Clinical Query in PubMed, to identify previous systematic reviews on the subject. The solitary review retrieved addressed several HRQoL instruments, had a broad review question and a limited search strategy [6].

A structured search was performed on March 10, 2014 in five electronic general databases: Cochrane Central, PubMed Medline, OvidSP Medline, OvidSP Embase and Thomson Reuters ISI Web of Knowledge Web of Science. The database searches were done from the year 2009 onwards as this was the year that the CAT was developed. We used a variety of search terms, including text words and database-specific subject headings, for articles in English, French or Spanish. The detailed, database-specific search strategies can be found in the online supplementary material. Top-ranked respiratory journals and the ProQuest Dissertations \& Theses database were manually searched. Reference lists from existing narrative reviews of the CAT were searched for potential studies, as were bibliographies of all included studies.

\section{Study selection, data collection process and data items}

Two reviewers (Nisha Gupta and Lancelot M. Pinto) independently screened the title and abstract of each study identified from the search. All the potentially relevant articles were then retrieved in full-text form and two reviewers (Nisha Gupta and Lancelot M. Pinto) performed the secondary screen after a review of the 
full text of the chosen articles. Disagreement on the inclusion or exclusion of a specific study was resolved by reaching a consensus. When necessary, disagreements were resolved by a third reviewer (Jean Bourbeau). A list of excluded studies and reasons for their exclusion was maintained.

Data were electronically extracted from each eligible study using a piloted data extraction form. The form was revised and improved after pilot data extractions were performed to assess concordance between the reviewers.

Two reviewers (Nisha Gupta and Andreea Morogan) independently extracted data from each included study, including study characteristics, population characteristics, interventions and/or events and outcomes studied, along with the corresponding measures of test performance. The data extraction form can be found in the online supplementary material.

\section{Quality assessment of included studies}

Two reviewers (Nisha Gupta and Andreea Morogan) independently performed quality assessment for each study using the Consensus-Based Standards for the Selection of Health Measurement Instruments (COSMIN) checklist, which is a validated quality assessment tool that evaluates the methodological quality of studies assessing psychometric properties of an instrument; it is the only specific checklist for methodological evaluation of psychometric properties on patient-reported outcomes [7, 8]. The methodological quality of each psychometric property was evaluated through a number of items and was scored using the four-point rating scale of "excellent", "good", "fair" and "poor". An overall score for the methodological quality of a study was given for each psychometric property by taking the lowest rating of an item ("worst score counts" method) [7]. The list of items, scoring rules and psychometric properties to which the checklist applies to can be found in the online supplementary material.

\section{Synthesis of results}

A narrative synthesis was employed to summarise the current knowledge on the CAT's reliability, validity, responsiveness and MCID. Data were tabulated through detailed tables that compared the studies with respect to study characteristics, population characteristics and CAT psychometric properties. Data synthesis was based on the provision of appropriate and similar outcomes studied, noting the specific statistical tests used. Computing a range (minimum to maximum) of study results on a particular psychometric property assessed the strength and adequacy of the psychometric property. A discussion of the impact of methodological quality on study results was explored to provide some assessment of quality and heterogeneity between the studies. All analyses were performed using STATA 11 (Stata Corporation, College Station, TX, USA).

\section{Risk of bias across studies}

Language bias was assessed by retrieving citations from the search strategy with language filters (English, French and Spanish) and without, and was reported as an average across the five general databases according to the filtered citations as a percentage of the overall citations retrieved.

\section{Results}

Study selection

Figure 1 shows the study selection procedure and numbers of studies screened, assessed for eligibility and included in the review. A total of 36 articles were included in the qualitative synthesis.

\section{Study characteristics}

Table 1 summarises the study and population characteristics of the included studies according to the number of outcomes assessed: nine (25\%) studies assessed reliability (internal consistency and test-retest), $32(89 \%)$ studies assessed validity (concurrent, convergent, longitudinal and known groups validity), ten (28\%) studies assessed responsiveness and four (11\%) studies assessed MCID [5, 9-43].

The CAT was administered in 32 countries spanning Europe, North America, South America, Asia and Africa, with 17 studies published in 2012 and 11 studies published in 2013. Of the 36 studies, 16 were prospective cohorts and the remaining 20 were cross-sectional. Types of interventions or events evaluated in the prospective cohorts included pulmonary rehabilitation, onset of an acute exacerbation, recovery from an acute exacerbation and usual care; and the duration of follow-up ranged from 2 to 24 weeks. The number of participants ranged between 45 and 6469 with the percentage of female subjects between $0 \%$ and 64.4\%. The range for mean age was between 55.9 and 73.0 years and mean FEV1 between $38.7 \%$ and $98.0 \%$ predicted. 23 studies specified the number of individuals in GOLD grades. 


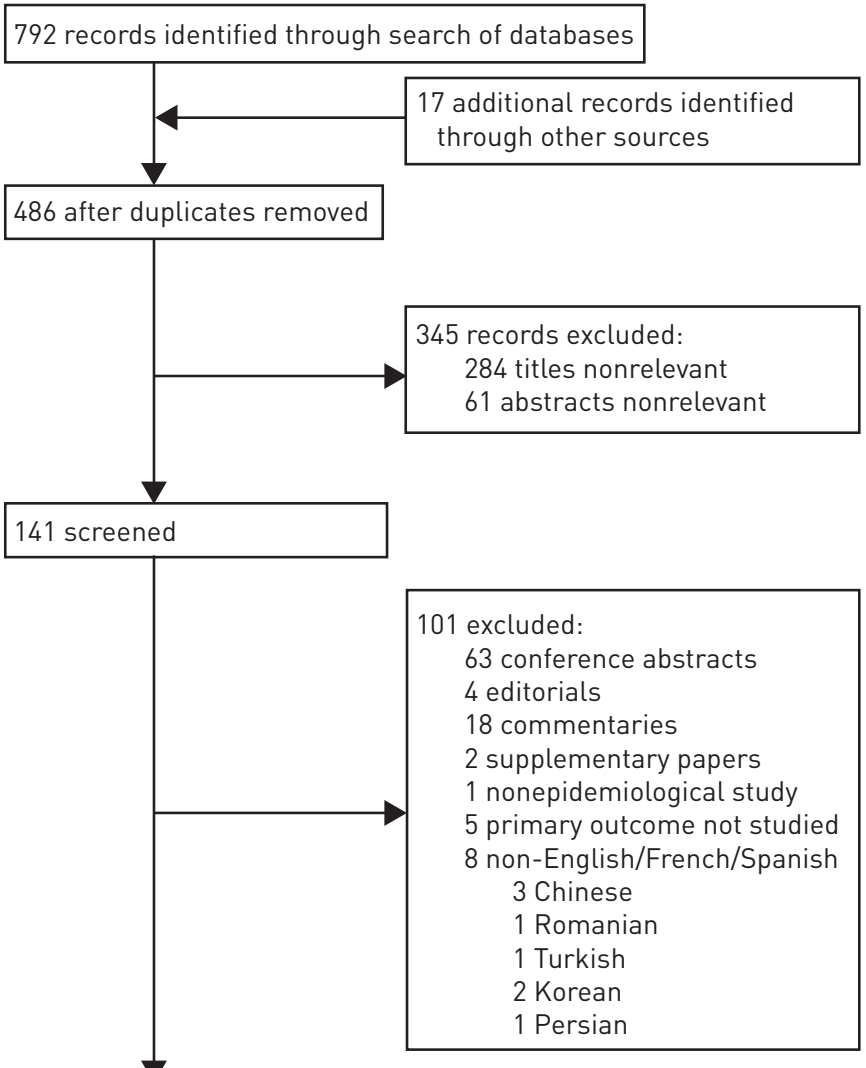

40 full-text articles assessed for eligibility

4 excluded:

4 primary outcome not studied

36 included in qualitative synthesis

FIGURE 1 Summary of literature search and study selection.

\section{Nonresponse rate and floor and ceiling effect}

Only six studies reported the proportions of the population with complete CAT scores (no missing items) and those with missing items $[9,15,17,21,26,41]$ (table 2). The percentage of patients with the minimum (floor effect) and maximum (ceiling effect) possible total score was measured in two populations $[9,36]$ (table 2).

\section{Reliability}

The internal consistency of the CAT was reported in eight studies, with the Cronbach's $\alpha$ range from 0.85-0.98 indicating a high correlation between items $[5,9,10,14,15,18,19,21]$ (table 2). Test-retest was evaluated in five studies to measure reproducibility $[5,9,10,14,30]$ (table 2), with the CAT administered on two different occasions (at baseline and then either 1 or 2 weeks later) for three studies $[5,10,30]$ and on three separate occasions (at baseline, 2 weeks and then 6 weeks later) for one study [14]. The ICC ranged from $0.80-0.96$, demonstrating that the CAT is consistent in producing scores when administered repeatedly under stable disease condition.

\section{Validity}

Three studies measured concurrent validity by comparing the total CAT score to healthcare utilisation $[15,34,39]$. The number of physician consultations was associated with total CAT scores in one study $(\mathrm{p}<0.001)$ [34], but not in the others $[15,39]$. The number of hospitalisations was directly associated with total CAT scores, irrespective of using the total CAT score $(\mathrm{p}<0.001)$ [15] or arbitrarily dichotomising the CAT score $($ e.g. $<10$ and $\geqslant 10)(p<0.001$ [34] and $\mathrm{p}<0.032$ [39]). Likewise, the number of emergency 


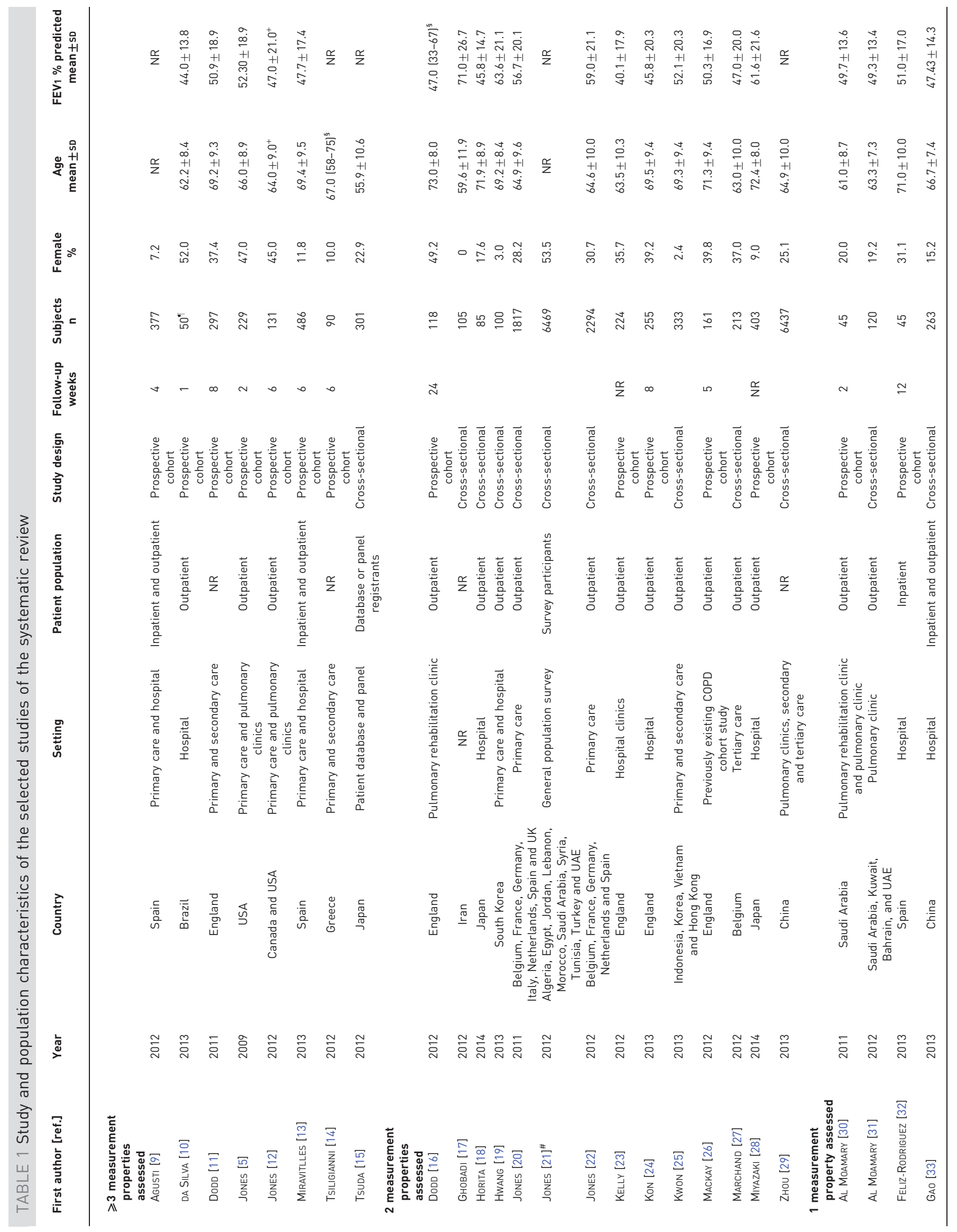




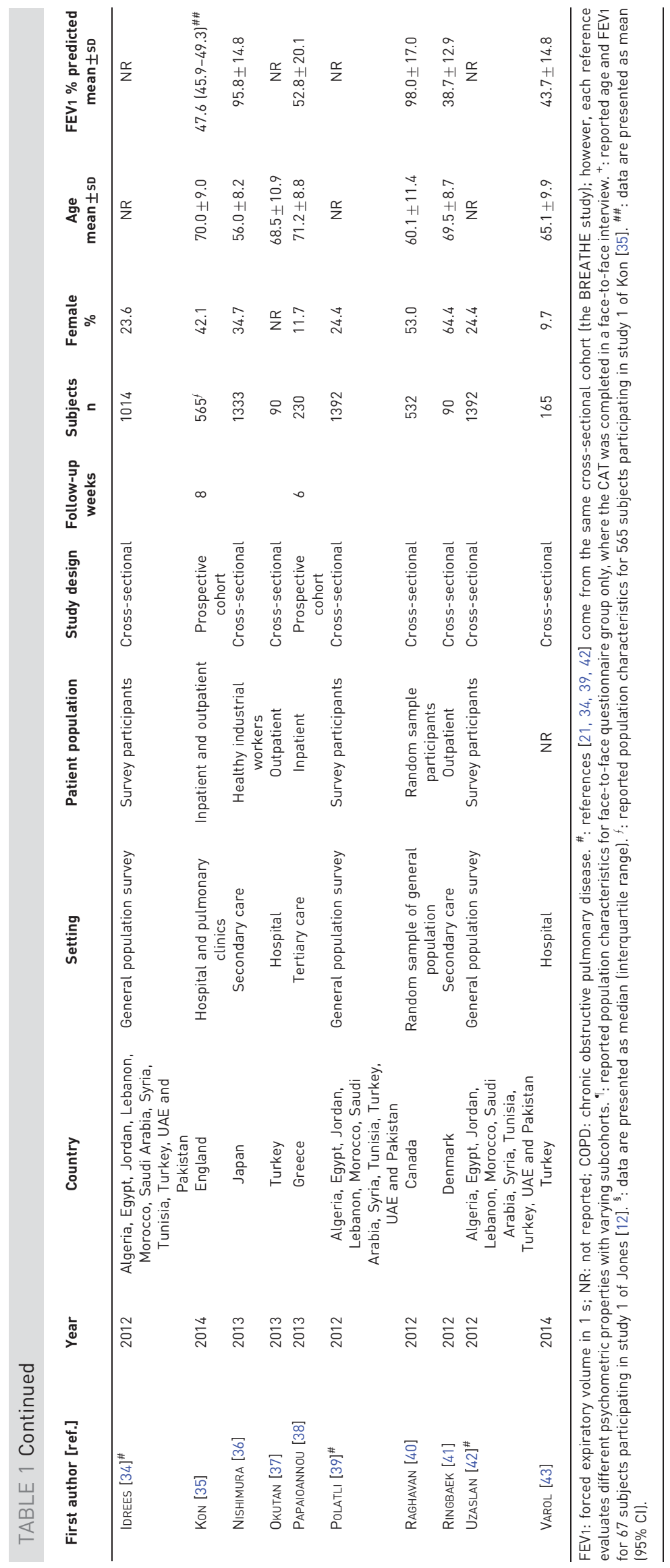


TABLE 2 COPD assessment test (CAT) nonresponse rate, floor and ceiling effect, internal consistency and test-retest reliability

Completed CATs and
missing items

\begin{tabular}{|c|c|c|c|c|c|c|}
\hline Agustı [9] & 99.7 & 0.3 & 377 & & & \\
\hline GноваDI [17] & 100 & 0.0 & 105 & & & \\
\hline JONES $[21]^{\#}$ & 99.3 & 0.7 & 6469 & & & \\
\hline MACKAY [26] & 98.3 & 1.7 & 161 & & & \\
\hline RINGBAEK [41] & 98.9 & 1.1 & 90 & & & \\
\hline TsudA [15] & 100 & 0.0 & 301 & & & \\
\hline Floor ${ }^{\top}$ and ceiling ${ }^{+}$effect & Floor effect \% & Ceiling effect \% & Subjects $n$ & & & \\
\hline AgustI [9] & 0.5 & 0.3 & 377 & & & \\
\hline NISHIMURA [36] & 0.0 & 7.6 & $145^{\# \#}$ & & & \\
\hline Internal consistency ${ }^{\S}$ & Cronbach's $\alpha$ & Subjects $\mathrm{n}$ & $\mathrm{p}$-value & & & \\
\hline Agustı [9] & 0.86 & NR & NR & & & \\
\hline DA SILVA [10] & 0.98 & 50 & 0.001 & & & \\
\hline HORITA [18] & 0.85 & 85 & NR & & & \\
\hline HWANG [19] & 0.85 & 100 & NR & & & \\
\hline JONES [5] & 0.88 & 1490 & NR & & & \\
\hline JONES [21] Arabic version & 0.85 & 4807 & NR & & & \\
\hline JONES [21] Turkish version & 0.86 & 1590 & NR & & & \\
\hline TSILIGIANNI [14] & 0.86 & 90 & NR & & & \\
\hline TsudA [15] & 0.89 & 301 & NR & & & \\
\hline \multirow[t]{2}{*}{ Test-retest ${ }^{f}$} & \multirow[t]{2}{*}{ ICC } & \multirow[t]{2}{*}{$\mathbf{n}$} & \multicolumn{3}{|c|}{ Administration duration weeks } & \multirow[t]{2}{*}{ p-value } \\
\hline & & & 1 & 2 & 3 & \\
\hline Agusti [9] & 0.83 & NR & NR & NR & NR & $N R$ \\
\hline Al MoAmary [30] & 0.90 & 45 & 0 & 2 & NA & 0.00008 \\
\hline DA SILVA [10] & 0.96 & 50 & 0 & 1 & NA & $0.93-0.97^{\bullet \oplus}$ \\
\hline JONES [5] & 0.80 & 53 & 0 & 1 & NA & NR \\
\hline TSILIGIANNI [14] & 0.94 & 90 & 0 & 2 & 6 & $0.92-0.96$ \\
\hline \multicolumn{7}{|c|}{ 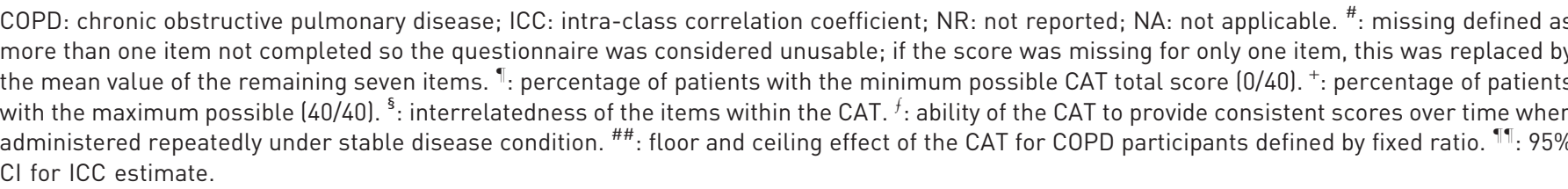 } \\
\hline
\end{tabular}

room visits was associated with the total CAT scores in two studies $(\mathrm{p}<0.001$ [15] and $\mathrm{p}<0.001$ [34]), but not in the other [39].

Convergent validity was assessed in 21 studies in which the CAT was compared to various questionnaires $[5,9-15,17-20,22,23,25,27-29,31,41,42]$ (table 3). The patients were in stable state when measuring this property unless it was unreported in the studies. CAT longitudinal validity was reported in six studies and the interventions or events consisted of pulmonary rehabilitation or recovery from an acute exacerbation $[9,11-13,16,24]$ (table 3).

19 studies reported known groups validity and the categories that could differ in HRQoL varied [5, 9, 11, $14,17,20-23,25-29,33,36,37,40,43$ ] (table 4). The CAT score was statistically different $(\mathrm{p}<0.05)$ in the following categories: COPD GOLD grades $[9,14,17,20,22,25,27,37]$; primary care physician-rated COPD GOLD grades [20, 22]; healthy individuals versus individuals diagnosed with COPD [21, 28, 33, 36, 40]; infrequent exacerbators versus frequent exacerbators (defined as no acute exacerbation in the last 6 months versus acute exacerbation in the last 6 months; $0-1,2-4$ or $>4$ exacerbations per year; and $<2$ or $\geqslant 2$ exacerbations per year) $[9,23,26,33,43]$; exacerbation state versus stable state $[5,9,20]$; body mass index (BMI) (defined as BMI $<18.5 \mathrm{~kg} \cdot \mathrm{m}^{-2}$, BMI $\geqslant 18.5$ and $<23 \mathrm{~kg} \cdot \mathrm{m}^{-2}$, or BMI $\geqslant 23 \mathrm{~kg} \cdot \mathrm{m}^{-2}$ ) [25]; and mMRC score $[9,27,37]$. The CAT score was not statistically different $(p \geqslant 0.05)$ in the following categories: sex [11, 20, 21, 29]; age (defined as $\leqslant 65$ years versus $>65$ years) $[20,29]$; current smokers versus nonsmokers [23]; and comorbidities (defined as $0,1-2$ or $\geqslant 3$ comorbidities) [20, 25]. 
TABLE 3 COPD assessment test (CAT) convergent and longitudinal validity

Instrument

Studies $\mathbf{n}$

Convergent validity

Longitudinal validity ${ }^{\Uparrow}$ with study details

\begin{tabular}{|c|c|c|c|c|}
\hline $\begin{array}{l}\text { Pearson's } \\
\text { correlation } \\
\text { range }\end{array}$ & $\begin{array}{l}\text { Spearman's } \\
\text { correlation } \\
\text { range }\end{array}$ & $\begin{array}{l}\text { Pearson's } \\
\text { correlation } \\
\text { range }\end{array}$ & Type of intervention/event & $\begin{array}{c}\text { Duration } \\
\text { weeks }\end{array}$ \\
\hline
\end{tabular}

\section{Disease-specific questionnaires \\ SGRQ-C}

$\mathrm{CCQ}$

SGRQ

CRQ

General quality of life

questionnaires

SF-36 (general health)

SF-12 (physical component)

SF-12 (mental component)

SF-6D

Clinical and physiological

measures

mMRC

6MWT

FEV1 \% predicted

$\begin{array}{cc}7 & 0.69-0.82 \\ 4 & \text { NA } \\ & 0.68-0.78 \\ 5 & 0.72-0.74 \\ 5^{\S} & -0.48--0.33\end{array}$

0.58

$-0.60$

$-0.34$

$-0.53$

$0.29-0.61$
$-0.37--0.27$
$-0.55--0.17$

$\begin{array}{cc}0.64 & 0.63 \\ \text { NA } & 0.60 \\ 0.64-0.76 & 0.13 \\ 0.65-0.84 & 0.36 \\ \text { NA } & -0.50--0.38\end{array}$

NA

$-0.65$

$-0.58$

NA
Exacerbation recovery

Exacerbation recovery

Pulmonary rehabilitation

Pulmonary rehabilitation

Pulmonary rehabilitation
4

6

8

8

$6-8$

COPD: chronic obstructive pulmonary disease; SGRQ: St George's Respiratory Questionnaire; SGRQ-C: SGRQ for COPD; CCQ: Clinical COPD Questionnaire; CRQ: Chronic Respiratory Questionnaire; SF: Short Form; mMRC: modified Medical Research Council dyspnoea scale; 6MWT: 6-min walk test; FEV1: forced expiratory volume in $1 \mathrm{~s} ; \mathrm{NA}$ : not applicable. ${ }^{\#}$ : correlation between the total CAT score and the score of another instrument that measures a similar construct. ": correlation between the change in total CAT score and the change in score of another instrument over time with an intervention/event. ${ }^{\S}$ : JoNES [12], Kon [24] and AL MOAMARY [31] had individual CRQ domain scores correlated to the total CAT score; therefore, individual correlations of the different domains were averaged to give a total $\mathrm{CRQ}$ correlation to the total CAT score.

\section{Responsiveness}

10 studies examined responsiveness of the CAT (table 5) [9, 11-14, 16, 24, 26, 32, 38]. The CAT was responsive to pulmonary rehabilitation in four studies and the range of mean change in CAT score was $-3.0--2.2$ units at the end of the intervention $[11,12,16,24]$. The majority of patients improved with pulmonary rehabilitation, whether it lasted for 8 weeks [11, 16, 24] or 6 weeks [12]. Responsiveness was reassessed from the end of an 8-week intervention to 6 months later, and it was determined that the total CAT score deteriorated slightly from the end of rehabilitation [16].

The majority of patients' total CAT score deteriorated with onset of an exacerbation: mean CAT score increased by 4.7 units $(\mathrm{p}<0.001)$ [26]. However, with exacerbation recovery on treatment, patients' CAT score improved over 2, 4, 6 or 12 weeks $[9,12,13,32,38]$ (table 5). Patients with and without depressive symptoms improved their CAT scores with exacerbation recovery, but those patients without depressive symptoms had greater improvement in CAT scores over 6 weeks [38].

\section{Minimum clinically important difference}

Four studies attempted to determine the MCID of the CAT [11, 12, 14, 35]. Three studies employed the anchor-based approach to calculate MCID. Of those, two studies found that their range of external responses (e.g. much better, a little better, no different or a little worse; responders or nonresponders) was not used by an equal proportion of patients; therefore, it was not possible to determine the MCID [11, 12]. The other study identified a decrease of 2 units as an MCID estimate [35]. Two studies used the distribution-based approach and determined an MCID for the CAT of 3.76 units [14] and a decrease that ranged from 3.3 to 3.8 units [35].

\section{Risk of bias within and across studies}

The methodological quality of the studies was mostly rated fair (30 (83\%) studies), with one rated poor, four rated good and one rated excellent, according to the COSMIN checklist. Internal consistency, testretest and convergent and known groups validity were evaluated in studies of fair and good methodological 
TABLE 4 COPD assessment test (CAT) known groups validity

\begin{tabular}{|c|c|c|c|c|c|c|}
\hline Agusti [9] & $12.4 \pm N R$ & $14.4 \pm N R$ & $20.9 \pm N R$ & $20.9 \pm N R$ & & 0.01 \\
\hline JONES [20] & $16.2 \pm 8.8$ & $16.3 \pm 7.9$ & $19.3 \pm 8.2$ & $22.3 \pm 8.7$ & & $0.0001^{\top}$ \\
\hline JONES [22] & $15.9 \pm 8.6$ & $16.5 \pm 8.0$ & $19.2 \pm 8.1$ & $22.4 \pm 9.0$ & & $0.0001^{\top}$ \\
\hline KWon [25] & $16.5 \pm N R$ & $16.0 \pm N R$ & $19.0 \pm N R$ & $21.3 \pm N R$ & & 0.001 \\
\hline MARCHAND [27] & $14.3 \pm N R$ & $16.6 \pm N R$ & $19.1 \pm N R$ & $23.7 \pm N R$ & & $0.0001^{\top}$ \\
\hline Healthy individuals versus COPD & No COPD & COPD & & & & p-value \\
\hline GAO [33] & $4.0 \pm 2.1$ & $10.0 \pm 5.3$ & & & & 0.001 \\
\hline JoNES [21] Turkey & $8.1(7.6-8.6)^{\S}$ & $20.9(19.6-22.2)^{\S}$ & & & & 0.0001 \\
\hline JONES [21] Arabic countries & $5.4(5.2-5.6)^{\S}$ & $16.6(15.5-16.8)^{\S}$ & & & & 0.0001 \\
\hline Agusti [9] & $15.8 \pm 8.1$ & $22.4 \pm 8.4$ & & & & 0.01 \\
\hline JONES [5] & $16.0 \pm N R$ & $20.7 \pm N R$ & & & & 0.0001 \\
\hline JONES [20] & $17.2 \pm 8.3$ & $21.3 \pm 8.4$ & & & & 0.0001 \\
\hline mMRC score & 0 & 1 & 2 & 3 & 4 & $p$-value \\
\hline Agusti [9] & $8.7 \pm N R$ & $14.8 \pm N R$ & $18.3 \pm N R$ & $24.9 \pm N R$ & $28.1 \pm N R$ & 0.01 \\
\hline MARCHAND [27] & NA & $12.7 \pm \mathrm{NR}^{f}$ & $19.9 \pm N R$ & $25.5 \pm N R$ & $25.2 \pm N R$ & 0.0001 \\
\hline OKUTAN [37] & $7.7 \pm 2.2$ & $13.9 \pm 6.1$ & $21.7 \pm 5.9$ & $27.5 \pm 2.2$ & $27.3 \pm 9.7$ & $0.021^{\# \#}$ \\
\hline
\end{tabular}

Data are presented as subcategory total CAT score mean \pm SD, when available from the study, unless otherwise stated. COPD: chronic obstructive pulmonary disease; GOLD: Global Initiative for Chronic Obstructive Lung Disease; mMRC: modified Medical Research Council dyspnoea scale; NR: not reported; NA: not applicable. " : ability of the CAT to differentiate scores between subcategories known to vary on health status; ": GOLD I and GOLD II are not significant; ${ }^{+}$: GOLD II, GOLD III and GOLD IV are not significant; ${ }^{\S}$ : data are presented as mean (95\% CI); ${ }^{f}$ : total CAT score for 0-1 mMRC score subcategory; ${ }^{\# \#}$ : mMRC scores 2,3 and 4 are not significant.

quality; longitudinal validity and responsiveness were examined in studies of fair methodological quality; MCID was evaluated in studies of fair and excellent methodological quality; and concurrent validity was assessed in studies of poor and fair methodological quality. Language bias was minimal across the five general databases; English, French and Spanish filtered citations as a percentage of the overall citations retrieved were 95.4\%.

\section{Discussion}

The goal in designing a HRQoL tool is for it to accurately and reliably measure HRQoL, and this review identifies the CAT's adequacy as a HRQoL instrument. Although several articles have been published on the CAT, this is the first study to systematically review the available literature evaluating the CAT's psychometric properties in a defined population of patients with COPD.

The psychometric properties of the CAT are both acceptable and favourable. The CAT is reliable: the interrelatedness of the eight items within the questionnaire indicate high internal consistency, while the stability of CAT total scores after repeated administrations confirms its reproducibility over time. Furthermore, the CAT demonstrates good construct validity through convergent, longitudinal and known groups validity. It is evident that the CAT is responsive and able to detect a change in score over time: the CAT score improved with pulmonary rehabilitation and exacerbation recovery on treatment, and the CAT score deteriorated with the onset of an exacerbation. Only one study reliably identified a decrease of 2 units as the MCID estimate through an anchor-based approach.

The majority of the studies (83\%) did not report missing data on the CAT, and in some studies only subjects with complete data were analysed, leading to a significant proportion of patients being excluded. Future research needs to examine the floor and ceiling effects of the CAT, as they have only been addressed in two populations. Overall, the methodological quality of the studies was rated fair. All of the 
TABLE 5 COPD assessment test (CAT) responsiveness ${ }^{\#}$ to pulmonary rehabilitation and exacerbation recovery on treatment

\begin{tabular}{|c|c|c|c|c|c|c|}
\hline \multirow[t]{2}{*}{ Study } & \multirow{2}{*}{$\begin{array}{c}\text { Duration } \\
\text { weeks }\end{array}$} & \multirow[t]{2}{*}{ Study defined groups } & \multicolumn{3}{|c|}{ CAT score } & \multirow[t]{2}{*}{ p-value } \\
\hline & & & Pre & Post & Change & \\
\hline \multicolumn{7}{|c|}{ Pulmonary rehabilitation } \\
\hline Dodd [11] & 8 & NA & $20.5 \pm 7.4$ & $17.5 \pm 7.7$ & $-2.9 \pm N R$ & 0.001 \\
\hline Dodo [16] & 8 & NA & $22.1 \pm 7.5$ & $19.2 \pm 7.6$ & $-2.9 \pm N R$ & 0.001 \\
\hline JONES [12] & 6 & NA & $17.9 \pm 6.5$ & $15.7 \pm 6.9$ & $-2.2 \pm N R$ & 0.002 \\
\hline Kon [24] & 8 & NA & $21.8 \pm 7.2$ & $18.8 \pm 7.4$ & $-3.0 \pm N R$ & 0.001 \\
\hline \multicolumn{7}{|c|}{$\begin{array}{l}\text { Exacerbation recovery on } \\
\text { treatment }\end{array}$} \\
\hline \multirow[t]{6}{*}{ AgustI $[9]^{+}$} & 2 & Much better & NR & NR & $-8.9 \pm 9.1$ & NR \\
\hline & & Considerably improved & NR & NR & $-4.8 \pm 6.0$ & NR \\
\hline & & Slightly improved & NR & NR & $-4.6 \pm 4.7$ & NR \\
\hline & & No change & NR & NR & 0 & NR \\
\hline & & Slightly worse & NR & NR & $1.6 \pm N R$ & NR \\
\hline & & Quite a lot worse & NR & NR & $4.7 \pm N R$ & NR \\
\hline FELIZ-RodRIGUeZ [32] & 12 & NA & $22.8 \pm 4.9$ & $15.6 \pm 4.5$ & $-6.5 \pm 3.9$ & 0.001 \\
\hline JONES [12] & 4 & NA & $21.4 \pm 7.7$ & $19.9 \pm 7.7$ & $-1.4 \pm 5.3$ & 0.03 \\
\hline MiRAVITLLES [13] & 6 & NA & $22.0 \pm 7.0$ & $12.1 \pm 5.9$ & $-9.9 \pm 5.1$ & 0.001 \\
\hline \multirow[t]{2}{*}{ PAPAIOANNOU [38] } & 6 & Depressive symptoms & $N R$ & $N R$ & $-7.0 \pm N R$ & 0.012 \\
\hline & & No depressive symptoms & NR & NR & $-11.0 \pm N R$ & 0.012 \\
\hline
\end{tabular}

Data are presented as mean $\pm S D$, when available from the study, unless otherwise stated. COPD: chronic obstructive pulmonary disease; NA: not applicable; NR: not reported. " : ability of the CAT to detect a change in score over time between interventions. ": negative change in CAT score is an improvement in CAT score (or health-related quality of life (HRQoL)); positive change in CAT score is a deterioration in CAT Score (or HRQoL). ${ }^{+}$: categories of change determined based on the HRQoL transition.

psychometric properties, except concurrent validity, were assessed in studies rated fair, good or excellent methodological quality.

Although the diversity of the studies retrieved resulted in examination of psychometric properties in many COPD populations, allowing for generalisation of the results, further assessment of validity and responsiveness needs to be completed in specific patient populations (e.g. females, younger age groups, mild disease) to assess the CAT's capability of discriminating between these groups. It would be of great utility to evaluate the predictive validity of the CAT to determine whether it can predict future clinical outcomes (e.g. mortality, hospital admission, disease progression or exacerbation). Similarly, while there is no correct manner to determine the MCID, several studies must attempt to provide estimates, so that multiple results can be combined to provide a true value (see online supplementary material for methods of calculating MCID). Moreover, linked to the development of the questionnaire was a grading system based on the CAT score, for which the development group proposed potential management considerations according to each scenario. An investigation of the impact of the CAT on the quality of the primary care consultations in patients with COPD has been conducted in a randomised controlled trial, although research needs to be advanced in this area due to the study's methodological limitations [44].

Strengths of this review include exhaustive search strategies across multiple databases, independent study retrieval, screening, data extraction and assessment of study quality. Data were insufficient to perform a meta-analysis: the variety of outcomes studied, methodological heterogeneity and diverse study populations prevented the generation of a common summary effect of a specific psychometric property, so a metaanalysis was deemed inappropriate.

There were, however, limitations. Investigating heterogeneity was not possible, but heterogeneity between studies is to still be expected. Likewise, formal assessments evaluating publication bias through a funnel plot could not be conducted. Although no language filters were applied in the search strategy, an assessment of the language bias indicated that a minor language bias could be of concern; however, the findings appear appropriate given that the CAT's development was studied in certain languages (e.g. English) but not in others. Limitations of the data from the included studies must also be considered (e.g. standard deviations for known groups validity were not presented in the majority of studies), although they reflect the dearth of literature available on the CAT. 


\section{Conclusion}

This review employed rigorous methodology to provide a comprehensive overview of the CAT's psychometric properties in patients with COPD. The studies support the reliability and validity of the CAT and that the tool is responsive to interventions, although the MCID remains debatable. Since the CAT demonstrates good performance and is a simple and quick tool that assesses the HRQoL in patients with $\mathrm{COPD}$, there is a growing interest in its use in clinical practice. Studies are needed to evaluate the use of this questionnaire for the symptomatic assessment of patients with COPD in the new GOLD classification. It cannot be assumed that the CAT behaves similarly with different patient population characteristics; thus, studies must also attempt to determine the validity of the CAT in females, patients with mild disease or individuals at risk, and younger and older patients.

\section{References}

1 Vestbo J, Hurd SS, Agusti AG, et al. Global strategy for the diagnosis, management, and prevention of chronic obstructive pulmonary disease: GOLD executive summary. Am J Respir Crit Care Med 2013; 187: 347-365.

2 Global Initiative for Chronic Obstructive Lung Disease (GOLD). Global Strategy for the Diagnosis, Management, and Prevention of COPD. Revised 2011.

3 Guyatt GH, Feeny DH, Patrick DL. Measuring health-related quality of life. Ann Intern Med 1993; 118: 622-629.

4 Jones P, Harding G, Wiklund I, et al. Improving the process and outcome of care in COPD: development of a standardised assessment tool. Prim Care Respir J 2009; 18: 208-215.

5 Jones PW, Harding G, Berry P, et al. Development and first validation of the COPD assessment test. Eur Respir J 2009; 34: 648-654.

6 Weldam SW, Schuurmans MJ, Liu R, et al. Evaluation of quality of life instruments for use in COPD care and research: a systematic review. Int J Nurs Stud 2013; 50: 688-707.

7 Terwee CB, Mokkink LB, Knol DL, et al. Rating the methodological quality in systematic reviews of studies on measurement properties: a scoring system for the COSMIN checklist. Qual Life Res 2012; 21: 651-657.

8 Mokkink LB, Terwee CB, Patrick DL, et al. The COSMIN study reached international consensus on taxonomy, terminology, and definitions of measurement properties for health-related patient-reported outcomes. $J$ Clin Epidemiol 2010; 63: 737-745.

9 Agusti A, Soler JJ, Molina J, et al. Is the CAT questionnaire sensitive to changes in health status in patients with severe COPD exacerbations? COPD 2012; 9: 492-498.

10 da Silva GP, Morano MT, Viana CM, et al. Portuguese-language version of the COPD assessment test: validation for use in Brazil. J Bras Pneumol 2013; 39: 402-408.

11 Dodd JW, Hogg L, Nolan J, et al. The COPD assessment test (CAT): response to pulmonary rehabilitation. A multicentre, prospective study. Thorax 2011; 66: 425-429.

12 Jones PW, Harding G, Wiklund I, et al. Tests of the responsiveness of the COPD assessment test following acute exacerbation and pulmonary rehabilitation. Chest 2012; 142: 134-140.

13 Miravitlles M, Garcia-Sidro P, Fernandez-Nistal A, et al. Course of COPD assessment test (CAT) and clinical COPD questionnaire (CCQ) scores during recovery from exacerbations of chronic obstructive pulmonary disease. Health Qual Life Outcomes 2013; 11: 147.

14 Tsiligianni IG, van der Molen T, Moraitaki D, et al. Assessing health status in COPD. A head-to-head comparison between the COPD assessment test (CAT) and the clinical COPD questionnaire (CCQ). BMC Pulm Med 2012; 12: 20.

15 Tsuda T, Suematsu R, Kamohara K, et al. Development of the Japanese version of the COPD assessment test. Respir Investig 2012; 50: 34-39.

16 Dodd JW, Marns PL, Clark AL, et al. The COPD assessment test (CAT): short- and medium-term response to pulmonary rehabilitation. COPD 2012; 9: 390-394.

17 Ghobadi H, Ahari SS, Kameli A, et al. The relationship between COPD assessment test (CAT) scores and severity of airflow obstruction in stable COPD patients. Tanaffos 2012; 11: 22-26.

18 Horita N, Yomota M, Sasaki M, et al. Evaluation of the chronic obstructive pulmonary disease assessment test in Japanese outpatients. Clin Respir J 2014; 8: 213-219.

19 Hwang YI, Jung KS, Lim SY, et al. A validation study for the Korean version of chronic obstructive pulmonary disease assessment test (CAT). Tuberc Respir Dis 2013; 74: 256-263.

20 Jones PW, Brusselle G, Dal Negro RW, et al. Properties of the COPD assessment test in a cross-sectional European study. Eur Respir J 2011; 38: 29-35.

21 Jones PW, Shahrour N, Nejiari C, et al. Psychometric evaluation of the COPD assessment test: data from the BREATHE study in the Middle East and North Africa region. Respir Med 2012; 106: Suppl. 2, S86-S99.

22 Jones PW, Brusselle G, Dal Negro RW, et al. Patient-centred assessment of COPD in primary care: experience from a cross-sectional study of health-related quality of life in Europe. Prim Care Respir J 2012; 21: 329-336.

23 Kelly JL, Bamsey O, Smith C, et al. Health status assessment in routine clinical practice: the chronic obstructive pulmonary disease assessment test score in outpatients. Respiration 2012; 84: 193-199.

24 Kon SS, Clark AL, Dilaver D, et al. Response of the COPD assessment test to pulmonary rehabilitation in unselected chronic respiratory disease. Respirology 2013; 18: 974-977.

25 Kwon N, Amin M, Hui DS, et al. Validity of the COPD assessment test translated into local languages for Asian patients. Chest 2013; 143: 703-710.

26 Mackay AJ, Donaldson GC, Patel AR, et al. Usefulness of the chronic obstructive pulmonary disease assessment test to evaluate severity of COPD exacerbations. Am J Respir Crit Care Med 2012; 185: 1218-1224.

27 Marchand E, Maury G. Évaluation du COPD assessment test (CAT) chez des patients BPCO en état stable [Evaluation of the COPD assessment test in patients with stable COPD]. Rev Mal Respir 2012; 29: 391-397.

28 Miyazaki M, Nakamura H, Chubachi S, et al. Analysis of comorbid factors that increase the COPD assessment test scores. Respir Res 2014; 15: 13.

29 Zhou QT, Mei JJ, He B, et al. Chronic obstructive pulmonary disease assessment test score correlated with dyspnea score in a large sample of Chinese patients. Chin Med J 2013; 126: 11-15. 
Al Moamary MS, Al-Hajjaj MS, Tamim HM, et al. The reliability of an Arabic translation of the chronic obstructive pulmonary disease assessment test. Saudi Med J 2011; 32: 1028-1033.

31 Al Moamary MS, Tamim HM, Al-Mutairi SS, et al. Quality of life of patients with chronic obstructive pulmonary disease in the Gulf Cooperation Council countries. Saudi Med J 2012; 33: 1111-1117.

32 Feliz-Rodriguez D, Zudaire S, Carpio C, et al. Evolution of the COPD assessment test score during chronic obstructive pulmonary disease exacerbations: determinants and prognostic value. Can Respir J 2013; 20 : e92-e97.

33 Gao Y, Hou Q, Wang H. Assessment of health status in patients with newly diagnosed chronic obstructive pulmonary disease. PLoS One 2013; 8: e82782.

34 Idrees M, Koniski ML, Taright S, et al. Management of chronic obstructive pulmonary disease in the Middle East and North Africa: results of the BREATHE study. Respir Med 2012; 106: Suppl. 2, S33-S44.

35 Kon SS, Canavan JL, Jones SE, et al. Minimum clinically important difference for the COPD assessment test: a prospective analysis. Lancet Respir Med 2014; 2: 195-203.

36 Nishimura K, Mitsuma S, Kobayashi A, et al. COPD and disease-specific health status in a working population. Respir Res 2013; 14: 61.

37 Okutan O, Tas D, Demirer E, et al. Evaluation of quality of life with the chronic obstructive pulmonary disease assessment test in chronic obstructive pulmonary disease and the effect of dyspnea on disease-specific quality of life in these patients. Yonsei Med J 2013; 54: 1214-1219.

38 Papaioannou AI, Bartziokas K, Tsikrika S, et al. The impact of depressive symptoms on recovery and outcome of hospitalised COPD exacerbations. Eur Respir J 2013; 41: 815-823.

39 Polatli M, Ben Kheder A, Wali S, et al. Chronic obstructive pulmonary disease and associated healthcare resource consumption in the Middle East and North Africa: the BREATHE study. Respir Med 2012; 106: Suppl. 2, S75-S85.

40 Raghavan N, Lam YM, Webb KA, et al. Components of the COPD assessment test (CAT) associated with a diagnosis of COPD in a random population sample. COPD 2012; 9: 175-183.

41 Ringbaek T, Martinez G, Lange P. A comparison of the assessment of quality of life with CAT, CCQ, and SGRQ in COPD patients participating in pulmonary rehabilitation. COPD 2012; 9: 12-15.

42 Uzaslan E, Mahboub B, Beji M, et al. The burden of chronic obstructive pulmonary disease in the Middle East and North Africa: results of the BREATHE study. Respir Med 2012; 106: Suppl. 2, S45-S59.

43 Varol Y, Ozacar R, Balci G, et al. Assessing the effectiveness of the COPD assessment test (CAT) to evaluate COPD severity and exacerbation rates. COPD 2014; 11: 221-225.

44 Gruffydd-Jones K, Marsden HC, Holmes S, et al. Utility of COPD assessment test (CAT) in primary care consultations: a randomised controlled trial. Prim Care Respir J 2013; 22: 37-43. 